stent against the previously placed external stents was performed (Figure 2,B). The TCPC was established on balloon deflation and removal (Figure 2, $C$ ). Angiography demonstrated a patent TCPC (Figure 2,D).

\section{Results}

All 4 animals survived and maintained normal sinus rhythm throughout both stages of the procedure. Successful TCPC was established in all animals without bleeding, stent displacement, or perforation.

\section{Discussion}

Sidiropoulos and colleagues ${ }^{2}$ reported a technique that combined a bidirectional cavopulmonary shunt and an intra-atrial lateral wall polytetrafluoroethylene baffle (Gore-Tex; W. L. Gore \& Associates, Inc, Flagstaff, Ariz) with multiple perforations to allow blood flow from the IVC into the atrium. The cardiac end of the SVC was subtotally banded. This method requires aortic crossclamping and IVC blood flow through the small perforations that might close while the patient awaits TCPC completion. Klima and colleagues ${ }^{3}$ reported experimental techniques, in which the unidirectional cavopulmonary shunt to the RPA was established off pump by means of a polytetrafluoroethylene graft and connected to the IVC through a self-expanding stent graft. Cheatham and associates ${ }^{4}$ and Murphy and coworkers ${ }^{5}$ have recently reported a similar approach by using a hemi-Fontan connection.

This new technique has the following advantages compared with previously described methods. It does not require cardiac arrest. A bidirectional cavopulmonary shunt can be established with the use of CPB but, if desired, without $\mathrm{CPB}$ aided by a temporary SVC-to-RA shunt. A fenestration of the intracardiac covered stent can be performed if needed. The stent graft could be dilated by using a catheter later in life to accommodate a patient's growth. The external caval stents are visible on fluoroscopy, providing an excellent marker for later percutaneous TCPC completion, as well as a buttress for the covered graft to expand within, allowing for a more reliable hemostatic seal. Finally, the IVC stent allows placement of the covered graft away from the hepatic veins.

\section{References}

1. Hausdorf G, Schneider M, Konertz W. Surgical preconditioning and completion of total cavopulmonary connection by interventional cardiac catheterization: a new concept. Heart. 1996;75:403-9.

2. Sidiropoulos A, Ritter J, Schneider M, Konertz W. Fontan modification for subsequent non-surgical Fontan completion. Eur J Cardiothorac Surg. 1998;13:509-12.

3. Klima U, Peters T, Peuster M, Hausdorf G, Haverich A. A novel technique for establishing total cavopulmonary connection: from surgical preconditioning to interventional completion. J Thorac Cardiovasc Surg. 2000;120:1007-9.

4. Cheatham JP, Galantowicz M, Torres W, Tower AJ, Hill SL, Kleinman $\mathrm{CS}$, et al. The use of custom-made covered NuMed CP stents in the treatment of congenital heart disease [abstract]. Catheter Cardiovasc Interv. 2002;57:100.

5. Murphy JD, Murdison KA, Nehgme RA, Pizzaro C, Norwood WI. Catheter facilitated completion of Fontan procedure [abstract]. Circulation. 2003;108(suppl IV):704.

\title{
Transsternal transpericardial approach for acute descending necrotizing mediastinitis
}

\author{
Franco Stella, MD, PhD, and Francesco Petrella, MD, Bologna, Italy
}

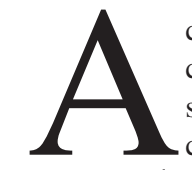

cute mediastinitis is a severe infection of mediastinal connective tissue between the 2 pleural cavities and surrounding median thoracic structures. It is usually caused by esophageal perforations or poststernotomy infections. ${ }^{1}$ Descending necrotizing mediastinitis (DNM) is one of

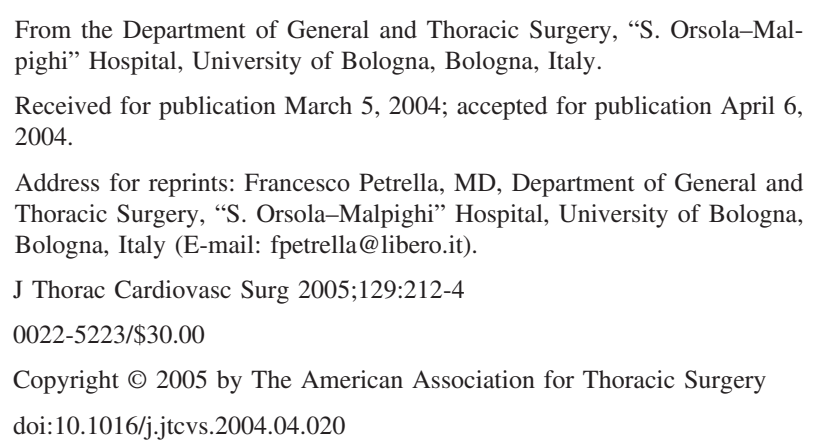

the most dangerous mediastinal infections and is caused by odontogenic or cervicofascial infections or cervical trauma. Infection descends along the deep cervical fascial space, causing cellulitis, necrosis, and abscess formation in the mediastinum, leading to sepsis. Hasegawa and coworkers ${ }^{2}$ proposed classifying DNM into 3 groups on the basis of infection extension: type I, infection localized in the upper mediastinum above the tracheal bifurcation and not always requiring aggressive mediastinal drainage; type IIA, infection extending to the lower anterior mediastinum; and type IIB, infection extending to the anterior and lower posterior mediastinum and demanding complete mediastinal drainage.

Only small series of DNM have been reported in recent literature, with mortality rates of between $25 \%$ and $40 \%$ in different series. $^{3}$ Toilette, debridement, drainage of infected fluid collections, and necrotic tissue exeresis are the surgical gold standard therapy, but the best surgical approach for this operation remains controversial.

We report a case of acute DNM after left parapharyngeal abscess that was treated through a transsternal transpericardial 

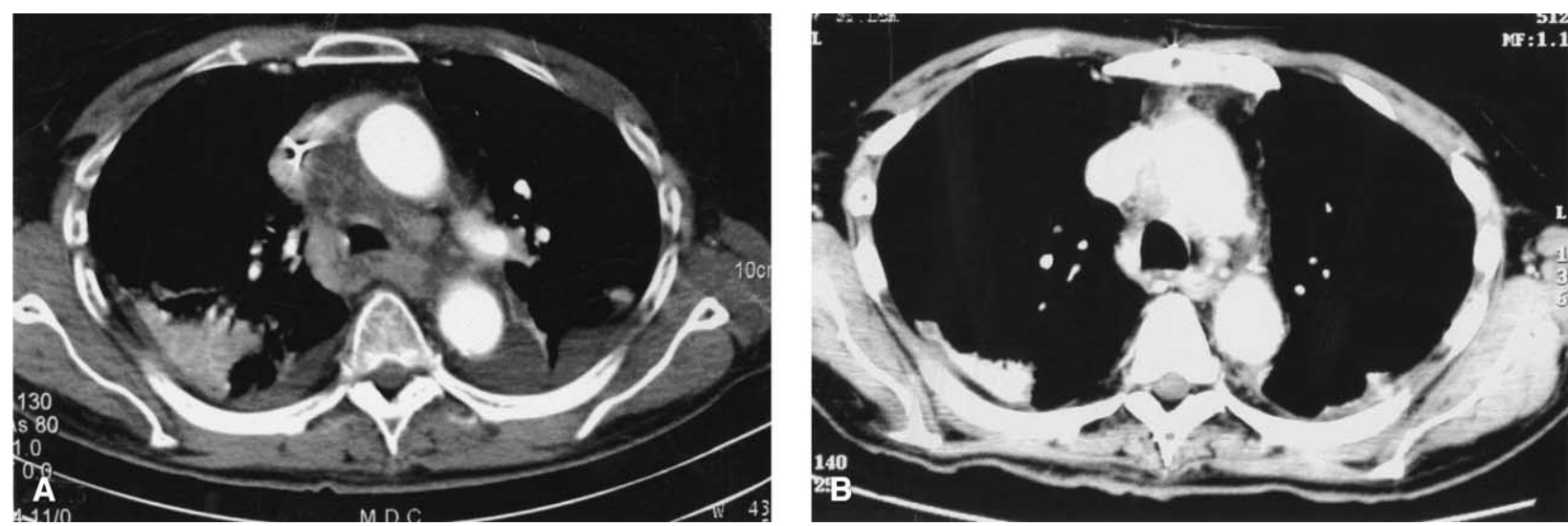

Figure 1. A, Preoperative CT scan. B, Postoperative CT scan.

approach, which offered a very good surgical field and allowed radical drainage and necrosectomy of infected tissue. The postoperative course was uneventful, and the patient was discharged 22 days after the operation.

\section{Clinical Summary}

A 76-year-old man was admitted to the emergency department because of 2 days of persistent fever $\left(38^{\circ} \mathrm{C}\right)$ unamenable to common broad-spectrum antibiotic therapy. The patient was dyspneic and asthenic. Physical examination showed bilateral laterocervical lymphoadenomegaly; laryngoscopy disclosed a left parapharyngeal collection filling the piriform sinus and partially occupying the laryngeal aditus, with respiratory space narrowing and left cord fixity. The otolaryngologist incised and drained the collection, determining a good recovery of respiration space. Admission tests showed a hemoglobin level of $12.9 \mathrm{~g} / \mathrm{dL}$ and a white blood cell count of 10,200 U/L. Neck and chest computed tomography (CT) scanning was performed and disclosed a retrolaryngeal prevertebral ovoid lesion, probably an abscess collection with medium contrast uptake, causing left posterolateral compression from the mandible to the hyoid. A left paratracheal mediastinal hypodense contrast enhancing mass was also disclosed that descended downward and completely surrounded the trachea and carina, replacing mediastinal fat tissue. Chest CT scan also showed bilateral pleural effusion (Figure 1, A).

We observed a sharp increase in fever, dyspnea, and septic state development not responding to broad-spectrum antibiotic therapy, and therefore we decided to operate. We performed median longitudinal sternotomy: the anterior pericardial foil was incised, and then the superior vena cava and aorta were mobilized and retracted to incise the posterior pericardial foil vertically. We then entered the retrocardiac mediastinal space, paying attention to the left recurrent nerve and esophagus (Figure 2). A large purulent effusion of that space was evacuated, and nondiagnostic microbiologic tests were carried out. Mediastinal toilette with iodate solution was performed, followed by debridement and necrosectomy. Six mediastinal Redon drainages on suction, 2 conventional mediastinal drainages, and 2 double-lumen pleural drainages were inserted. The sternotomy was conventionally closed with stainless-steel stitches. The postoperative period was uneventful. The orotracheal tube was removed 4 days after the operation, postoperative CT scanning was performed (Figure 1, B), and the patient was discharged 22 days after the operation.

\section{Discussion}

DNM is an acute polymicrobic infection of the mediastinum originating from an oropharyngeal or cervical infection. The infective and necrotizing process might descend into the mediastinum, pleural cavities, pericardium, and abdomen through the contiguous deep spaces of the neck as entry portals. ${ }^{4}$ DNM might cause abscess, empyema, pleuropericardial effusion, intrathoracic hemorrhage, and cardiac tamponade with a fatal outcome. The high mortality rate of DNM is due to diagnostic uncertainty and to the difficulty of creating an adequate continuous mediastinal drainage after toilette and necrosectomy. 5,6

Estrera and colleagues ${ }^{7}$ first described surgical therapy for DNM. They claimed that mediastinal drainage should be necessarily performed after thoracic access when necrotic infection spreads under the fourth thoracic vertebra posteriorly or the tracheal bifurcation anteriorly. In all the other cases they deemed transcervical mediastinal drainage alone to be sufficient.

Subsequently, many other authors advocated a transthoracic approach with thorough mediastinal exploration irrespective of the infection level because of the fast evolution of the necrotizing process. ${ }^{8}$

Surgical intervention is currently the gold standard therapy for DNM resistant to antibiotic therapy. However, a number of surgical approaches have been described, including the subxiphoideal approach, the clamshell incision, median sternotomy, or the thoracoscopic approach. Posterolateral thoracotomy has long been considered the best approach to DNM because the prevertebral and paraesophageal spaces are well exposed without the risk of osteomyelitis attributed to sternotomy. Thoracotomy, however, does not permit contralateral mediastinal toilette, necrosectomy, or exploration of the contralateral pleural cavity very often involved with pleural effusion, and therefore in some cases involving global mediastinal DNM and circumferential tracheal involvement, as in our case, posterolateral thoracotomy is inadequate.

Anterior mediastinotomy with or without subxiphoideal drainage has been proposed for anteriorly localized mediastinitis be- 

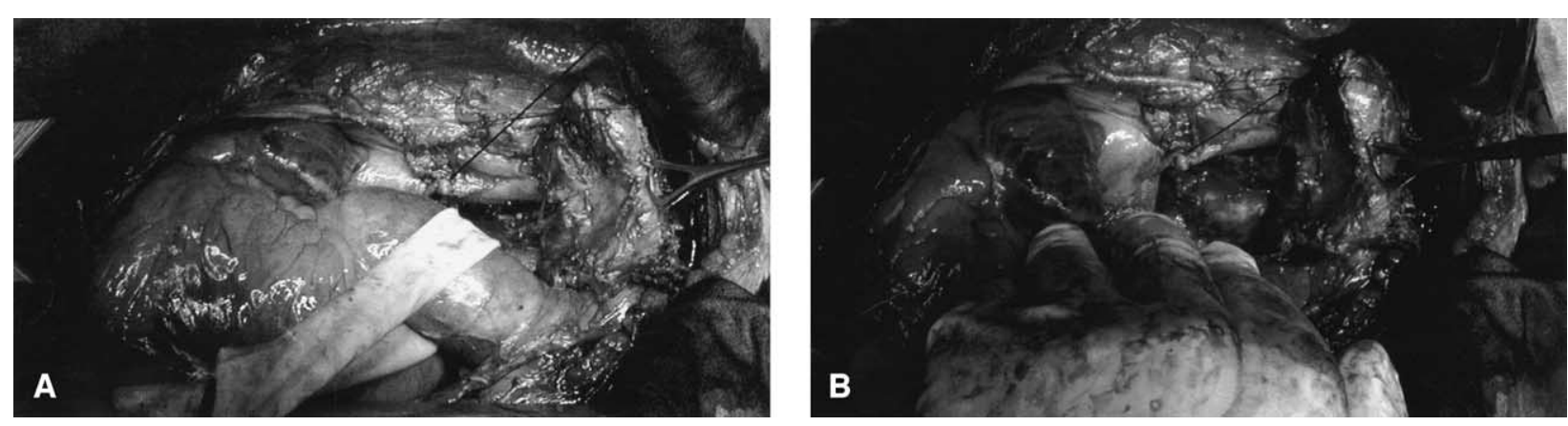

Figure 2. A, Intraoperative view: step 1. B, Intraoperative view: step 2.

cause of the low invasiviness of the surgical procedure. However, this approach does not offer a good global operative field and is contraindicated in a posteriorly developing necrotizing process, as in our patient.

Ris and coworkers ${ }^{8}$ described DNM surgical therapy using the clamshell approach. This technique offers a wide surgical field, allowing bilateral exploration of the pleural cavities. They discouraged longitudinal sternotomy because of the high risk of sternal osteomyelitis and dehiscence, especially when no additional muscle or omental flaps are interposed between the debrided mediastinum and the transected sternum. In our view the clamshell incision carries the same risk of osteomyelitis as longitudinal sternotomy because of sternum transection. In addition, this approach is extremely aggressive, requiring double thoracotomy and transverse sternotomy and strongly influencing the mechanical ventilatory activity of already compromised patients. There is also a risk of phrenic nerve hyperextension and mono or bilateral diaphragmatic paralysis.

The transsternal transpericardial approach for selected cases of DNM offers more benefits than risks. This approach allows complete mediastinal exploration, whereas the thoracotomy, transcervical, or subxiphoidal approaches preclude such a thorough exploration. The clamshell incision offers a very good surgical field, especially for the left pleural cavity, but it is extremely aggressive and much more dangerous than sternotomy in such critical patients.

The osteomyelitis risk after sternotomy for mediastinitis has often been emphasized. Our surgical approach, together with multiple drainages, is commonly used for mediastinitis after cardiac surgery. Redon drainages with a high suction system $(-700 \mathrm{~mm} \mathrm{Hg})$, together with traditional mediastinal drainages $\left(-20 \mathrm{~cm} \mathrm{H}_{2} \mathrm{O}\right.$ ), allow continuous mediastinal toilette in the presternal space, avoiding bone involvement by the mediastinal infective process. The gradual retraction and then removal of mediastinal drainages offer a very good control system in the postoperative period, and the characteristics of Redon drainages (small size, rigidity, and high suction capacity) will cleanse even dead spaces in the mediastinum. ${ }^{9}$ We usually use from 5 to 9 Redon drainages and 1 or 2 traditional mediastinal drainages, depending on the aggressiveness of infection and chest dimensions.

In conclusion, despite problems such as vascular dissection, the transsternal transpericardial approach is a useful and effective technique for the surgical treatment of selected patients with DNM refractory to broad-spectrum antibiotic therapy.

\section{References}

1. Papalia E, Rena O, Oliaro A, Cavallo A, Giobbe R, Casadio C, et al. Descending necrotizing mediastinitis: surgical management. Eur J Cardiothorac Surg. 2001;20:739-42.

2. Hasegawa T, Endo S, Sohara Y. Classification of descending necrotizing mediastinitis. Ann Thorac Surg. 2000;69:1296.

3. Freeman RK, Vallieres E, Verrier ED, Karmy-Jones R, Wood DE. Descending necrotizing mediastinitis: an analysis of the effects of serial surgical debridement on patient mortality. J Thorac Cardiovasc Surg. 2000;119:260-7.

4. Melero Sancho LM, Minamoto H, Fernandez A, Ubirajara Sennes L, Riscegli Jatene F. Descending necrotizing mediastinitis: a retrospective surgical experience. Eur J Cardiothorac Surg. 1999;16:200-5.

5. Marty-Ane CH, Alauzen M, Alric P, Serres-Cousine O, Mary H. Descending necrotizing mediastinitis. Advantage of mediastinal drainage with thoracotomy. J Thorac Cardiovasc Surg. 1994;107:55-61.

6. Marty-Ane CH, Berthet JP, Alric P, Pegis JD, Rouvière P, Mary H. Management of descending necrotizing mediastinitis: an aggressive treatment for an aggressive disease. Ann Thorac Surg. 1999;68: 212-7.

7. Estrera AS, Lanay MJ, Grisham JM, Sinn DP, Platt MR. Descending necrotizing mediastinitis. Surg Gynecol Obstet. 1983;157:545-52.

8. Ris HB, Banic A, Furrer M, Caversaccio M, Cerny A, Zbaren P. Descending necrotizing mediastinitis: surgical treatment via clamshell approach. Ann Thorac Surg. 1996;62:1650-4.

9. Durandy Y, Batisse A, Bourel P, Dibie A, Lemoine G, Lecompte Y. Mediastinal infection after cardiac operation. A simple closed technique. J Thorac Cardiovasc Surg. 1989;97:282-5. 